\title{
Evolved navigation theory and the descent illusion
}

\author{
Russell E. JaCkson and LaWrence K. Cormack \\ University of Texas, Austin, Texas
}

\begin{abstract}
Researchers often assume that height perception results from all of the same mechanisms as does other distance perception (Avraamides, Loomis, Klatzky, \& Golledge, 2004; Foley, Ribeiro-Filho, \& Da Silva, 2004; $\mathrm{Wu}$, Ooi, \& He, 2004). Evolved navigation theory (ENT) proposes that natural selection has differentiated some psychological processes, including height perception, in response to the navigational outcome of falling. We tested predictions from three theories in two experiments. Only ENT predicted greater height perceived from the top than from the bottom of a vertical surface (because descent results in falls more often than does ascent). Participants across experiments perceived an average of 32\% greater vertical distance when viewing from the top than when viewing from the bottom. We discuss selected implications and suggest ENT for uniting isolated findings, including the vertical-horizontal illusion.
\end{abstract}

Current research sometimes generalizes findings from largely horizontal distance-perception studies to all distance perception without considering that height perception may result from processes not shared with other distance perception (Avraamides, Loomis, Klatzky, \& Golledge, 2004; Foley, Ribeiro-Filho, \& Da Silva, 2004; $\mathrm{Wu}, \mathrm{Ooi}, \& \mathrm{He}, 2004)$. Does height perception result from all of the same processes as does other distance perception, or does it result from limited specialized processing? The former may appear more parsimonious, but we should answer empirical questions such as this experimentally.

Theorized mechanisms for specialized height perception include differential eye movements (Wundt, 1874, as cited in Winslow, 1933), differentially excitatory retinal meridians (Avery \& Day, 1969), and discord among retinal, visual, and gravitational orientations (Higashiyama, 1996). Peripheral mechanisms such as these may explain how we perceive heights, but they fail to explain why we might perceive heights differently from other distances. Although height perception is surely accomplished by some perceptual mechanism, only a causal theory can suggest why height perception might differ from other distance perception.

Two current causal theories of specialized height perception are foreshortening of receding horizontals and gravity theory. Segall, Campbell, and Herskovitz (1966) maintain that foreshortening of receding horizontals increases perceived vertical length in order to represent distance accurately, because vertical retinal surfaces can represent environmentally horizontal receding surfaces. Gravity theory (see Howard \& Templeton, 1966, p. 37) and other effort-based theories suggest that perceived distance corresponds with anticipated navigation effort. Gravity theory suggests that vertical surfaces should appear longer than horizontal surfaces because heights require greater energy to navigate than do horizontal surfaces.

\section{Evolved Navigation Theory}

Disparity between the modern environment and the environments in which humans evolved provides an important tool for behavior researchers (Jackson, 2004). In this article, we propose a broad evolutionary approach to investigating perceptual and navigational systems and then test a subset of predictions as they apply to height perception. Evolved navigation theory (ENT) suggests that physical and psychological navigation processes reflect natural selection from navigational costs reliably present over evolutionary time. ENT applied to height perception focuses on the major cost inherent in vertical or angled navigation: falling.

Falling is more common and costly on vertical surfaces than on horizontal ones. One might thus expect vertical surfaces to be processed differently than horizontal surfaces, in a way that incorporates this cost asymmetry when making navigational decisions. An effective mechanism that we know would decrease navigation of a costly surface would be to make the surface appear longer, because, intuitively and empirically, organisms pursue the nearer of otherwise equivalent navigational goals (Somervill \& Somervill, 1977). ENT applied to height perception suggests that specialized perceptual mechanisms could subvert preference for nearer goals by making vertical surfaces appear longer than horizontal ones. This height overestimation would facilitate horizontal navigation choices, when available, helping to avoid falling costs. If height perception in human ancestors contained genetic variance in accuracy, then some degree of overestimation likely incurred the least net cost, over time, because falling inflicts potentially severe costs. 
Falling injuries likely influenced reproductive fitness in all environments in which humans evolved. The most effective way to avoid falling costs would be to avoid completely those surfaces associated with falling. However, if height navigation did not always result in falls, and if it reliably held important resources, then height perception might instead weight navigational decisions by falling cost likelihood. What important resources might height navigation have offered reliably over evolutionary time?

Heights offer advantages similar to those suggested for human bipedalism (Conroy, 1997, p. 227) - namely, increased visibility and access to targets such as animal prey, plant resources, water, or other humans. Moreover, access to elevated surfaces, and the increased visibility that they afford, helps in forming large and detailed cognitive maps.

Second, geologic heights provide escape and defense from predators and other humans - a major cause of mortality over evolutionary time (Keeley, 1996). Ancestral humans navigated geologic heights such as hills, steppes, boulders, and cliffs within normal human ranges and in migrations leading to modern dispersal. Habitable heights offer the above benefits at night, when humans sleep and have reduced ability to see approaching threats. Habitation of heights has occurred in places as diverse as the Acropolis in Athens, Swayambunath in Nepal, and the Cliff Palace of Mesa Verde in Colorado.

Third, humans require large amounts of fresh water, which is often associated with steep surfaces (because flowing water erodes its substrate and water flows to the lowest of available surfaces). It is not uncommon that accessing sources of fresh water, and the food and materials located therein, requires vertical navigation. Access to the ocean and its associated food and materials also often requires vertical navigation.

Finally, trees offer access to arboreal prey, eggs, fruits, nuts, shelter, and material items such as wood and leaves. Arboreal species who share recent common ancestors with humans overestimate vertical in relation to horizontal distance (Dominguez, 1954).

Initial natural selection leading to the hypothesized height overestimation may have occurred phylogenetically prior to distinct humans - as suggested by the presence of vertical overestimation in nonhuman primates (Dominguez, 1954). The factors outlined above suggest at least maintenance selection for height overestimation in ancestral and modern humans. Prehuman ancestors surely interacted with the above-mentioned heights (and others) that just as likely selected for the onset of height overestimation - which selection could then maintain in humans, for the same reasons. Ultimately, height overestimation could persist in any derived lineage, even without current benefits, either because of the absence of sufficient genetic variation or because height overestimation inflicted insignificant cost. The root of ENT is that any species navigating surface orientations that reliably affect reproductive fitness might face selection for perceptual tendencies such as height overestimation. ENT asserts that humans either are, or are descended from, such organisms.

\section{One ENT Prediction: Falling Cost Asymmetry Across Position on Vertical Surface}

There is an inherent asymmetry in vertical navigation not present in horizontal navigation that distinguishes an ENT-derived approach from other theories. Ascending from the bottom and descending from the top of a height pose different costs. Ascending expends more energy than descending (Bassett et al., 1997; Minetti, Moia, Roi, Susta, \& Ferretti, 2002; Teh \& Aziz, 2002), but descending results in falls more often than ascending. Svanstrom (1974) found that falls on stairs occurred $76 \%$ of the time during descent. Tinetti, Speechley, and Ginter (1988) found that stepping down resulted in injurious falls among the elderly four times as often as stepping up (p. 1704). Haslam and Bentley (1999) found that postal workers fell while descending steps or inclined drives 15 times as often as when ascending (p. 39). Cohen and Lin (1991) found that ladder accidents occurred during descent nearly twice as often as during ascent (p. 31).

Body mechanics of descent increase its falling risk. Posture during descent requires leading with less dexterous feet, and it places the eyes posterior to the direction of travel, thereby reducing visibility. Whereas those ascending can keep their bodies close to the vertical surface, those descending must place their bodies out from the vertical surface in order to be able to see where they are going. This position results in poorer selection of holds, which (nonprehensile) feet cannot grip, while simultaneously positioning one's body farther from the surface, making it more difficult to retain grip than during ascent.

Descending inhibits control of velocity and direction of travel. Ascending necessitates muscle contractions that pull one's body weight to a specific point, but descending necessitates relaxation of muscles to allow partially controlled drops in the direction of gravity. This produces poorer control of velocity and poorer selection of hand and foot placement than does ascending.

Lack of experience on a height when initiating vertical movement also increases costs of falling more during descent than ascent. The beginning of an ascent or descent predicts success in traversing the surface. When ascending, one can predict ascent safety by testing handholds while holding oneself off the ground with one's feet, or by being able to fall a few inches relatively safely. When descending, however, one cannot predict safety by testing a surface, because the likeliest cost of failure is to fall the entire vertical length. The inability to practice descending forces greater certainty of success to guide the decision to descend than is the case with the decision to ascend.

\section{Predictions From Three Theories}

All of the following differential height perception theories argue against a viewpoint, which we title unitary distance perception, in which all distances are perceived via the same processes with similar outputs. We tested these theories by comparing participants' estimates of the height of a surface against its true vertical length. Specifically, we compared participants' estimates from the top of a vertical surface with height estimates from the bottom of the same surface. 
Foreshortening of receding horizontals (Segall, Campbell, \& Herskovits, 1966) predicts that we adjust perception of foreshortened surfaces in order to give exocentrically veridical distance estimates. Foreshortening of receding horizontals specifically predicts equal height estimation from both the top and the bottom of a vertical surface, as long as the actual distances are equal and are equally foreshortened.

Gravity theory (see Howard \& Templeton, 1966, p. 37) predicts overall height estimates exceeding the true height, because vertical surface navigation is more energetically expensive than other distance navigation. Gravity theory specifically predicts greater distance estimates from the bottom of a vertical surface than from the top, because ascending is more energetically expensive than descending.

Evolved navigation theory-derived predictions predict overall height estimates exceeding true height, because vertical navigation is associated with falling risk. These predictions specifically predict a descent illusion, in which height is overestimated more from the top of a vertical surface than from the bottom, because descent results in the costs of falling more than does ascent.

We tested predictions from each theory in two experiments, in which we used different methods and settings.

\section{EXPERIMENT 1}

Participants estimated an outdoor vertical surface length while positioned at its top and at its bottom, by approximating an equal horizontal length.

\section{Method}

Sixty-six participants from an introductory psychology course who reported normal (20:20) or corrected-to-normal vision met a research assistant (RA) in a campus office, completed a consent form, and answered a few screening questions. The questions screened for normal vision, body height, and fear of heights. The participants then proceeded to the outdoor testing site with the RA.

The participants estimated the height of a $14.39-\mathrm{m}$ vertical surface from both its top and its bottom, as illustrated in Figure 1. The RA randomly determined participant starting-position order (top first or bottom first) and then positioned participants near the same lateral point on the vertical surface for both estimates. Using a plainly worded script, the RA instructed the participants to estimate the distance from the top railing to the ground directly beneath it. The participants estimated vertical length by adjusting the distance from the RA to the vertical surface with hand signals; the RA then measured the distance back to the vertical surface with a wheeled measure.

In order to study this phenomenon in an ecologically valid setting, with rich stimuli, we modeled these procedures after previous outdoor distance-estimation research (Chapanis \& Mankin, 1967; Dixon \& Proffitt, 2002; Higashiyama, 1996; Higashiyama \& Ueyama, 1988; Yang, Dixon, \& Proffitt, 1999). Understandably, there exists some imprecision in the task and measurement under such conditions, but an important aspect of these techniques lies in the invariance between estimation procedures at the top and bottom positions. Predictions tested here focus on top- and bottom-estimate differences, and we used equivalent procedures between positions. We were not aware of any by-products of our methods that would artifactually alter distance judgments. We also implemented these techniques as precisely as possible via high selectivity in RA training and research site choice.

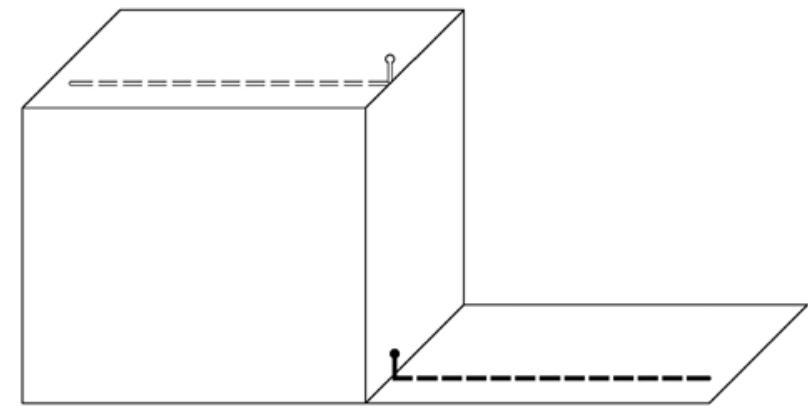

Figure 1. The Experiment 1 participant position during top (outline) and bottom (solid) estimates. Dotted icons represent the same participant during each estimate. Dashed lines represent the horizontal path in estimating the vertical height at each position. Figure not drawn to scale.

Author R.E.J. trained all RAs, who were not allowed to collect data until they made measurements within 1 in. $(2.54 \mathrm{~cm})$ of measurements by the author and other trained RAs. The measurement procedures were simple and specific, allowing accurate measurement with little room for interpretation. All of the RAs were blind to the experimental predictions and were observed unknowingly over the duration of the experiment to ensure that they followed all of the procedures precisely. No deviance from the designed techniques was observed.

The testing environment consisted of a parking garage selected for ecological validity to cues important to the theories under investigation. The height was great enough to inflict falling costs, and both participant positions were not impassably obstructed, such as with a window or screen. The participants could turn and lean freely and take as much time, and make as many adjustments, as they desired. Use of an elevator minimized arousal differences between estimates, and both top and bottom areas consisted of similar asphalt and cement surfaces.

\section{Results}

Figure 2 shows a scatterplot of participant height estimates in meters. Each data point in the figure plots the top estimate versus the bottom estimate for 1 participant. Arrows represent mean height estimate from the top ( $26.54 \mathrm{~m}, S D=6.83 \mathrm{~m})$ and the bottom $(20.61 \mathrm{~m}, S D=$ $4.65 \mathrm{~m})$. Top and bottom estimates correlated moderately highly $[r(66)=.609, p<.001]$. The dashed lines in each dimension represent actual height: $14.39 \mathrm{~m}$. Mean estimates exceeding the actual distance suggest height overestimation from both positions. Indeed, every participant overestimated height from the top. Not surprisingly, a manipulation check against true height indicates height overestimation from both the top $[t(65)=14.462, p<$ $.001]$ and the bottom $[t(65)=10.879, p<.001]$.

The solid line in Figure 2 represents the slope of unity between the top and bottom estimates. Most data points in this plot occur above the slope of unity, which illustrates the finding of most interest: Participants overestimated height more from the top than from the bottom of the vertical surface. In this case, 58 of the 66 participants overestimated the height more from the top position than from the bottom position. The binomial probability of this many (or more) data points falling above the slope of unity, if perceived height was actually the same, is roughly $9.0 \times 10^{-11}$. 


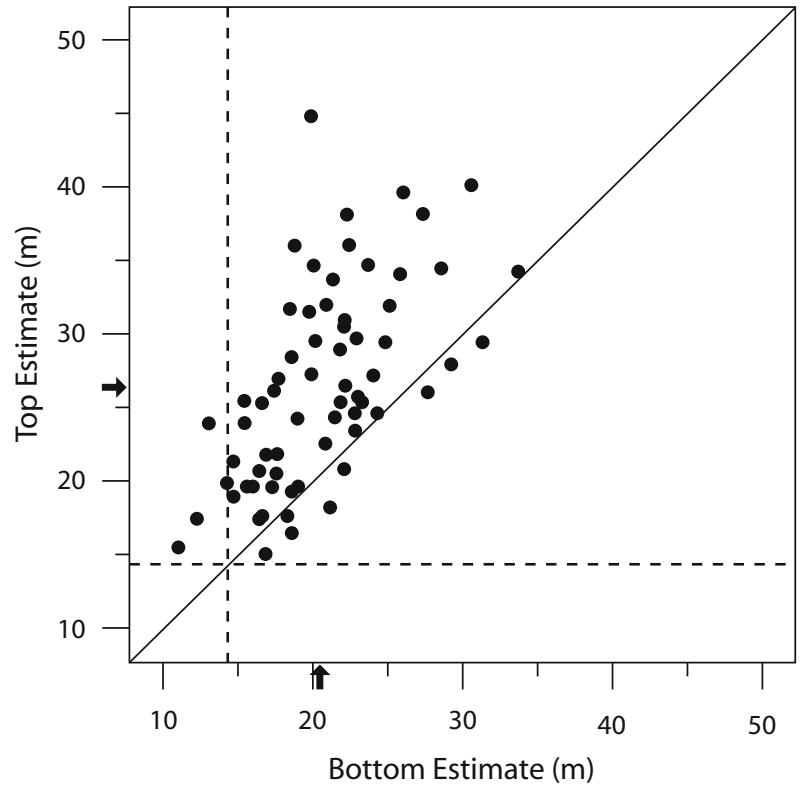

Figure 2. The Experiment 1 participant distance estimates, by position, in meters. Arrows indicate mean estimates from the top and the bottom. Dashed lines indicate actual vertical distance. The solid line indicates the slope of unity between the top and bottom estimates.

Figure 3 illustrates mean estimates by position, with error bars showing $95 \%$ confidence intervals. The dashed line in Figure 3 represents the true height. The mean height estimate from the bottom was $20.61 \pm 1.12 \mathrm{~m}$, whereas the mean height estimate from the top was $26.54 \pm 1.65 \mathrm{~m}$. The mean estimate was $29 \%$ greater at the top than at the bottom, with a difference of $5.93 \mathrm{~m}$. A $5.93-\mathrm{m}$ position difference between estimates of a 14.39 -m surface suggests a strong effect of position, with mean estimates at the top equaling $184 \%$ of the actual distance.

Not surprisingly, a paired samples $t$ test indicated a significant difference between participants' estimates of length from the top and from the bottom $[t(65)=8.865$, $p<.001]$. Participants perceived much greater vertical distance from the top of the height than from the bottom. These data support the primary ENT prediction and are inconsistent with predictions from unitary height perception and the two other differential height theories.

An independent samples $t$ test indicated that order of testing (i.e., beginning at the top or at the bottom) failed to influence top- and bottom-estimate differences $[t(64)=$ $1.019, p=.312]$. Significance tests of Pearson productmoment correlations failed to indicate that participant body height correlated with top- and bottom-estimate differences in men $[r(33)=-.099, p=.584]$ or women $[r(33)=.043, p=.813]$. Average estimate difference $(5.93 \mathrm{~m})$ exceeded even the greatest body height $(2.08 \mathrm{~m})$ by more than a factor of two. Neither order of testing nor body height changed participants' perception of greater height from the top than from the bottom.

We asked participants to rate their fear of heights in response to the question "Do you fear heights?" on a 5-point
Likert scale, with response options of not at all, slightly, moderately, very much, and intense, possibly irrational, fear. We were concerned about the chance that screening for participant height fear might lead participants to consider heights as more important during the experiment than they otherwise would have. However, we were unable to avoid asking about fear of heights, because we wanted to ensure that no acrophobic participants (characterized by responding with intense, possibly irrational, fear) participated and, as a result, experienced undue stress during our experiments. The extent to which screening for fear of heights influenced our methods appears minimal, because the participants were uninformed about the actual distances and the existence or position of upcoming estimates. We employed a double-blind method concerning our predictions, and testing order failed to have an impact on difference scores.

\section{Discussion}

Participants perceived greater height when standing at the top of a vertical surface than when standing at the bottom. These data are consistent with ENT-derived predictions, exclusive of predictions from the other causal height-perception theories. ENT suggests that costs of falling present in height navigation produced adaptive processes that we propose resulted in overestimation of specific geographical distances associated with falling: a descent illusion.

Although these data are consistent with ENT, we decided to expand this investigation with a second experiment. The first reason was simply to generalize; we wanted to make sure that nothing specific about the task or the testing site was responsible for the pattern of observed results.

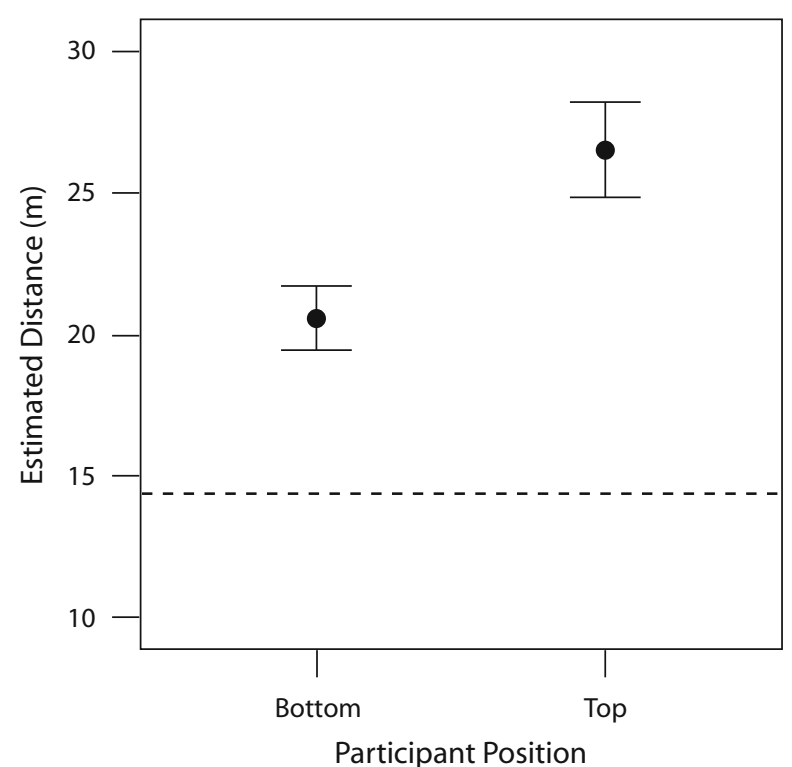

Figure 3. The Experiment 1 mean participant distance estimate, by position, in meters. Bars represent $95 \%$ confidence intervals about the means. The dashed line indicates actual vertical distance. 
Second, the monocular depth cue of familiar size might have mitigated the effect in Experiment 1. Since participants adjusted the distance from the same RA in both positions, familiar size could have biased estimates from both positions to be more similar than they would have been without a familiar size cue.

Finally, the distance from the participants' eyes to the plane of the vertical surface differed between the top and bottom positions in Experiment 1. The participants stood out from the surface by about $60 \mathrm{~cm}$ when making estimates from the bottom, because the vertical surface extended all the way to the ground. However, when viewing from the top, the participants' eyes were positioned $10-20 \mathrm{~cm}$ beyond the plane of the vertical surface. We therefore wanted to collect data in a setting in which the participants' eyes could be roughly the same distance from the vertical surface plane from both the top and bottom positions.

\section{EXPERIMENT 2}

In Experiment 1, participants estimated a fixed vertical interval via a variable horizontal interval. In Experiment 2, the participants estimated a fixed horizontal interval via a variable vertical interval. We also used a small, unfamiliar ball to denote distance, which presumably provided less of a familiar size cue than was used in Experiment 1 . Further, the study site in Experiment 2 included an overhang that allowed participant positioning directly beneath the vertical surface, more closely matching the viewing geometry from the top position (see Figure 4). This experiment allowed us to use an environment and methods different from those used in Experiment 1, for greater generalizability.

\section{Predictions From Three Theories}

The phenomenon of interest is identical between the two experiments, but predictions of which distances participants will perceive as greater are inverted from Experiment 1, because Experiment 2 participants matched a fixed horizontal with a variable vertical interval:

Foreshortening of receding horizontals predicts, as before, overall accurate and equal height estimates from the top and from the bottom.

Gravity theory predicts greater perceived height from the bottom than from the top, because ascending is more energetically expensive than descending. The participants should therefore match the fixed horizontal interval with a smaller physical vertical interval at the bottom than at the top.

Evolved navigation theory-derived predictions predict the same descent illusion of greater perceived height from the top than from the bottom, because descent results in falls more than does ascent. The participants should therefore match the fixed horizontal interval with a larger vertical interval at the bottom than at the top.

\section{Method}

One hundred forty-four participants from an introductory psychology course who reported normal (20:20) or corrected-to-normal vision met an RA in a campus office and completed the consent form and screening questions used in Experiment 1. The participants then proceeded to the outdoor testing site with the RA, who was trained in the same method used in Experiment 1.

For the testing site, we selected an outdoor campus staircase, for the reasons of ecological validity and experimental control stated in Experiment 1.

The participants estimated the length of a 7.20 -m horizontal distance from both the top and the bottom of the $15.90-\mathrm{m}$ vertical surface illustrated in Figure 4. The RAs did not tell the participants that the horizontal distance was equal in both positions. The RA randomly determined starting-position order (top first or bottom first). The RA then positioned participants at the same lateral point on the vertical surface for top and bottom estimates and verbally instructed the participants from a plainly worded script.

The participants' task was to estimate the horizontal distance between the vertical surface and a point $7.20 \mathrm{~m}$ away in both positions (denoted by a white line or a brick wall). The participants estimated the top horizontal distance by telling an RA standing next to them to lower or raise a black, white-speckled 4.5-cm-diameter rubber ball suspended from clear monofilament fishing line until the distance to the ball appeared equal to the horizontal length. The participants changed the distance to the ball while at the bottom position by showing an "up" or "down" sign to an RA at the top of the height. The starting position for the ball was at participant chest height in both positions. The RAs allowed the participants as much time and as many adjustments as they desired during estimation, then spooled in the fishing line and took an elevator to the next position. The RAs measured the participants' estimates after the testing session.

The RAs initially instructed the participants to estimate the distance between the ball and the ground for the bottom estimate and the distance between the ball and a chest-high railing for the top estimate. However, 3 of the first 15 participants verbally commented on their strong preference to use the distance from their eyes to the ball in both positions, so the RAs allowed those and all remaining participants to do so by describing this estimation. This participant preference was likely caused by the overhanging structure under which the

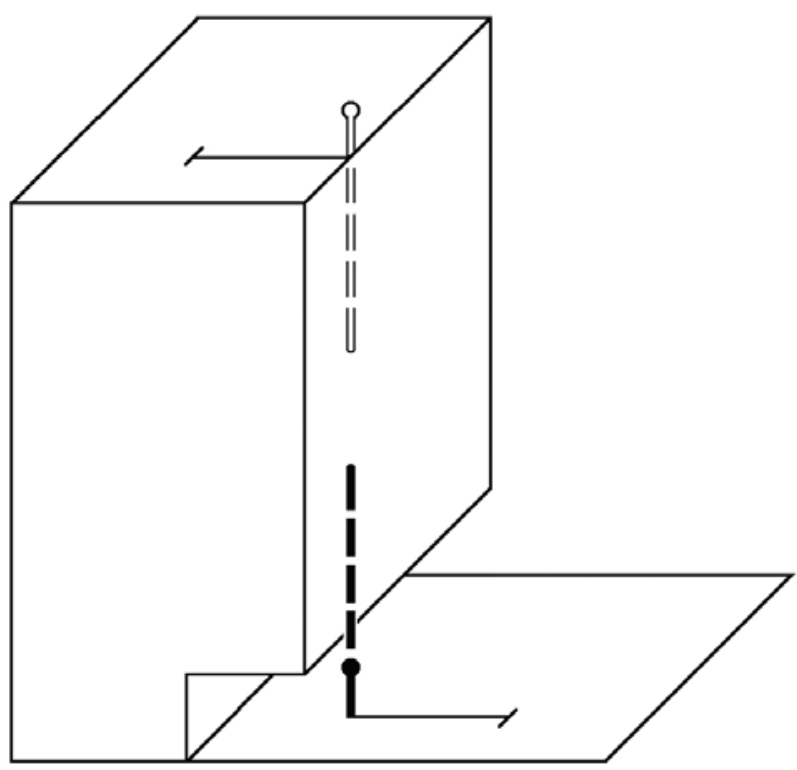

Figure 4. The Experiment 2 participant position during top (outline) and bottom (solid) estimates. Dotted icons represent the same participant during each estimate. Dashed lines represent the vertical path in estimating the horizontal distance for each position. The barred lines indicate the horizontal distance to estimate for each position. Figure not drawn to scale. 
participants stood for the bottom estimate. Positioning participants directly beneath the vertical surface necessitated a nearly $180^{\circ}$ head rotation, whereas participants in Experiment 1 regularly rotated their heads only roughly $90^{\circ}$.

We gathered additional data (the distance from the eyes to the surface of estimation) to reflect participants' desired estimates. We compiled this distance for all participants in both positions. Because the instructions initially suggested estimating from the ground or the railing, we will first report the results of participants' estimates as if they had used the ground when at the bottom and the railing when at the top, and then report participants' estimates from their eyes, in both positions.

\section{Results}

Estimates from the railing and from the ground. The mean estimate from the bottom position was $8.68 \mathrm{~m}$, $S D=1.31 \mathrm{~m}$, and from the top position it was $5.01 \mathrm{~m}$, $S D=1.30 \mathrm{~m}$. Although these results seem to confirm ENT predictions, the fact that (most) participants estimated the distance from their eyes, rather than from the ground or from the railing, would exaggerate the effect predicted by ENT. Thus, to be conservative and in order to reflect participants' intended estimates accurately, we corrected the data as follows. For estimates from the bottom, we subtracted a standard distance from the eyes to the top of the head $(15.24 \mathrm{~cm})$ from each individual participant's height and subtracted the resulting distance from estimates from the bottom. For estimates from the top, we added the average distance from the top railing to the participants' eyes while leaning over the railing $(30.48 \mathrm{~cm})$. These distances reproduced the distance from the eyes to the ball for each participant and replicated what (most) participants set as perceptually equal to the fixed horizontal interval.

Estimates from the eyes. Figure 5 shows a scatterplot of the data from Experiment 2, plotted in the same way as the data are plotted in Figure 2. Arrows represent the mean estimate of horizontal from the top $(5.31 \mathrm{~m}, S D=$ $1.30 \mathrm{~m})$ and from the bottom $(7.12 \mathrm{~m}, S D=1.28 \mathrm{~m})$. As in Experiment 1, the top and bottom estimates correlated $[r(144)=.222, p<.01]$. Dashed lines in each dimension represent the actual horizontal distance: $7.20 \mathrm{~m}$. Mean estimates less than the actual distance suggest height overestimation. A manipulation check against true horizontal distance indicates statistically significant height overestimation from the top $[t(143)=-17.411, p<.001]$ but not from the bottom (unlike in Experiment 1) $[t(143)=$ $-0.328, p=.744]$.

The solid line in Figure 5 represents the slope of unity between the top and bottom estimates. The ENT prediction suggested a descent illusion of greater height overestimation from the top than from the bottom of the vertical surface. Figure 5 would support this prediction if the majority of points fell below the slope of unity. In fact, 129 of the 144 points do fall below the slope of unity. An effect this large or larger would only occur with a binomial probability of $4.3 \times 10^{-24}$ if participants actually perceived height equally from the top and the bottom.

Figure 6 shows mean estimates by position, with error bars showing $95 \%$ confidence intervals about the means. The dashed line in Figure 6 represents true horizontal distance. The mean height estimate from the bottom was

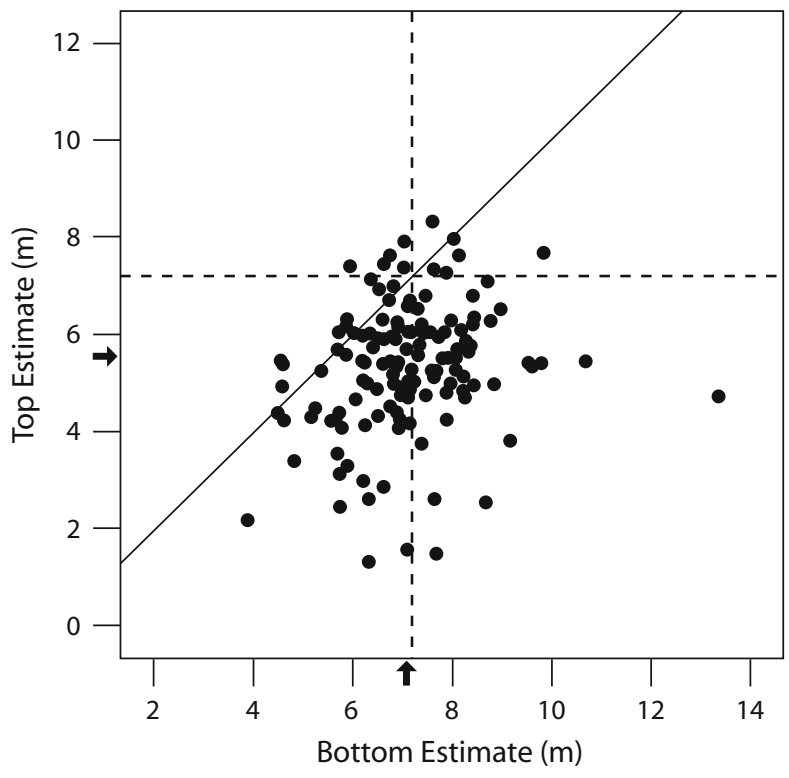

Figure 5. The Experiment 2 participant distance estimates, by position, in meters. Arrows indicate the mean estimates from the top and bottom. Dashed lines indicate the actual vertical distance. The solid line indicates the slope of unity between top and bottom estimates.

$7.12 \pm 0.21 \mathrm{~m}$, whereas the mean height estimate from the top was $5.31 \pm 0.21 \mathrm{~m}$. The mean estimate was $34 \%$ greater at the bottom than at the top, with a difference of $1.81 \mathrm{~m}$. A $1.81-\mathrm{m}$ position difference between estimates of a 7.20-m surface suggests a strong effect of position, with the actual distance constituting $136 \%$ of the mean estimate at the top.

Not surprisingly, a paired samples $t$ test indicated a significant difference between participants' estimates of length from the top and from the bottom $[t(143)=13.465$, $p<.001]$. Participants perceived much greater vertical distance from the top of the height than from the bottom, consistent with the descent illusion predicted by ENT.

An independent samples $t$ test suggested that order of testing (i.e., beginning at the top or at the bottom) failed to influence top and bottom estimate differences $[t(142)=$ $-.620, p=.536]$. Significance tests of Pearson productmoment correlations failed to suggest that participant body height correlated with top- and bottom-estimate differences in men $[r(77)=.044, p=.707]$ and women $[r(66)=-.006$, $p=.959]$ (1 participant failed to report sex). Neither order of testing nor body height changed participants' perceptions of greater height at the top than at the bottom.

\section{Discussion}

These data appear consistent with the descent illusion predicted by ENT, in which participants perceive greater distance from the top of a height than from the bottom. Additionally, these data suggest that participants overestimate height distance in comparison with horizontal distance when at the top of a height. ENT suggests that costs of falling present in height navigation could have produced an adaptive overestimation of specific geographi- 


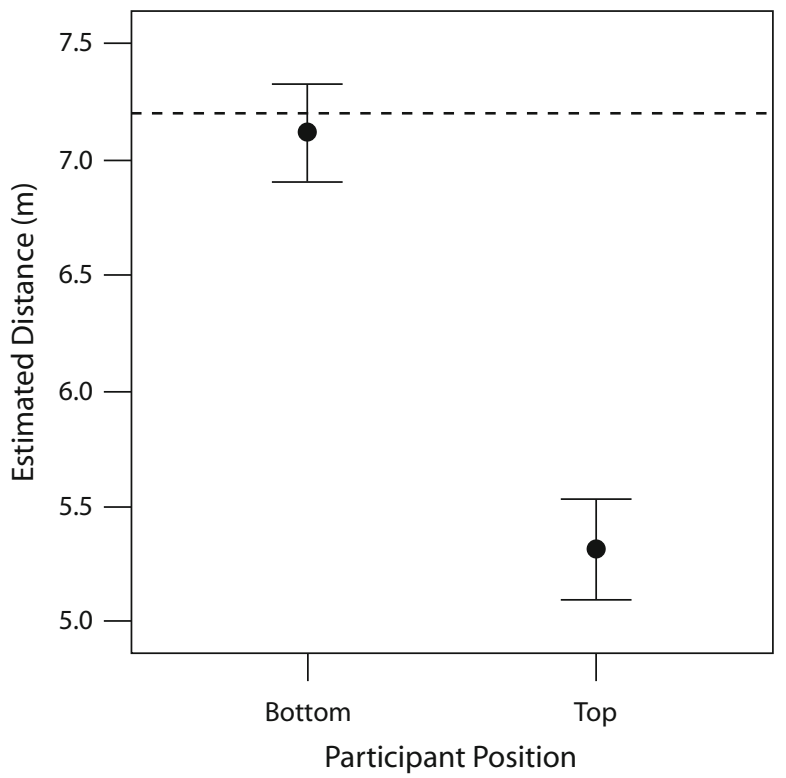

Figure 6. The Experiment 2 mean participant distance estimate, by position, in meters. Bars represent $95 \%$ confidence intervals about the means. The dashed line indicates actual horizontal distance.

cal distances associated with falling - a descent illusion, in this scenario.

Lack of height overestimation from the bottom position may have resulted artifactually from the testing site. We required participants to stand directly underneath the vertical surface, in order to better equate viewing conditions across positions. However, many participants wanted to move back, in order to have a more natural view of the vertical surface; this would have made their view more similar to that provided in Experiment 1. Mark, Jiang, Steinbach, and Paasche (1999) found a similar effect of viewing restrictions hampering distance estimates. Looking directly up, across a gap, may have reduced ecological validity of this particular method, because such an estimate would likely rarely occur in an ecologically valid setting - people would usually have additional views of a surface from farther away.

Data analyses suggested that restricting participants to estimate across the gap affected judgments. If we subtract the distance from the top of participants' heads to the bottom of the vertical surface at the bottom estimate $(M=6.23 \mathrm{~m}, S D=1.23)$, a $t$ test against the true distance $(7.20 \mathrm{~m})$ now suggests a significant height overestimation $[t(143)=-9.437, p<.001]$, and a paired samples $t$ test still suggests a significant difference between top and bottom estimates $[t(143)=-4.650, p<.001]$. These data, in conjunction with Experiment 1, may also reflect the finding that vertical extents are overestimated in proportion to their perceived size (Chapanis \& Mankin, 1967; Dixon \& Proffitt, 2002; Yang et al., 1999).

We allowed participants to use their eyes as the end reference point when making estimates, instead of the chest-high railing at the top and the ground at the bottom. However, the initial instructions likely influenced some participants. If we were able to subtract the estimates of individuals who used the instructed reference points to any degree, the mean top estimate would decrease and the mean bottom estimate would increase. This would broaden the disparity between top and bottom estimates and increase the effect of position observed in Experiment 2 data.

\section{GENERAL DISCUSSION}

We demonstrated a descent illusion in which participants overestimate vertical height much more when viewing it from the top than from the bottom. J. Stefanucci (personal communication, August 14, 2005) found similar results in recent work using different methods. Our participants in Experiment 1 perceived the vertical surface from the top as 1.84 times its true height, on average. The participants in Experiment 2 perceived the vertical surface from the top as 1.36 times its true height, on average. Expressed differently, the perceived height from the top averaged $32 \%$ greater than the perceived height from the bottom (29\% and 34\% in Experiments 1 and 2, respectively). Not only the average effects are large; we found some overestimation in 100\% (66 of 66) of participants in Experiment 1 and in 97\% (139 of 144) of participants in Experiment 2. These results illustrate a key ENT-derived prediction: that perceived surface height relates to potential navigation cost. We posit from ENT that the inherently greater risk of falling associated with descending than with ascending a vertical surface accounts for the asymmetrical descent illusion seen in these distance estimates.

We had participants estimate via distance matching. The key prediction compared estimates across the top and bottom positions; therefore, invariance across these two estimates is the most important quality of the procedures. Because our procedures did not differ across position, it is highly unlikely that anything related to the procedures per se could have produced the observed descent illusion. We know of no procedure that would produce the magnitude of effects found in these data by chance, and similar distance-matching procedures have been used widely in similar distance estimates (Chapanis \& Mankin, 1967; Dixon \& Proffitt, 2002; Higashiyama, 1996; Higashiyama \& Ueyama, 1988; Yang et al., 1999). Further, Da Silva and Dos Santos (1984) have shown that a variety of distance estimate techniques produce very similar results.

An obvious question raised by these experiments is why the size of the effect, expressed as a percentage of the actual distance estimated, varied across the two experiments for estimates from both locations. Can we assume that a perceptual distortion such as the overestimation of heights would behave like a meridional magnification, so that objects would appear expanded along the vertical axis? This would result in an effect similar to wearing cylindrical (astigmatic) lenses. The data are inconsistent with this simple idea, however.

When we compare Experiment 1 with Experiment 2, the distance to estimate decreased from $14.39 \mathrm{~m}$ to $7.20 \mathrm{~m}$. This corresponded to overestimation from the top decreasing from a factor of 1.84 to 1.36 (i.e., from an $84 \%$ 
to a $36 \%$ overestimate), and overestimation from the bottom decreasing from 1.43 to 1.01 (i.e., from a $43 \%$ to a $1 \%$ overestimate). Although an analogy to magnification is seemingly parsimonious, no a priori reason suggests that it is the form that an adaptation to falling costs would take. Whereas meridional magnification is trivial to implement in an optical system, there is unlikely to be a simple neural analogue to a cylindrical lens in a system as distributed as the human visual system.

Furthermore, data suggest that vertical extents are associated with greater overestimation on the basis of perceived size, not meridional magnification. Yang et al. (1999) found that participants overestimated a vertical line (of PVC tubing) in natural scenes to a greater percentage when viewing the real stimuli outdoors $(12 \%$ overestimation) than when viewing photographs ( $2 \%$ overestimation) or drawings ( $3 \%$ overestimation) of the same objects. The authors suggest that this outcome results from greater perceived object size when in the environment than when viewing pictures or line drawings. Yang et al. subsequently ran additional experiments in which they confirmed that overestimation (expressed as a percentage) increased with the perceived object size. Chapanis and Mankin (1967) also demonstrated this effect. These studies suggest that participants overestimate vertical distances relative to perceived object size. Here and elsewhere (Jackson \& Cormack, 2006a, 2006b, 2006c, 2006d), we suggest that perceived size may determine overestimation only insofar as size approximates likely falling costs.

Differences between our two experiments are consistent with greater vertical extents inducing greater perceptual distortion as a proxy for falling cost. Distance estimate differences between top and bottom positions were roughly the same (about $40 \%$ ) across both experiments. Figure 7 illustrates that overestimation from the top, expressed as a relative change from the estimate from the bottom, is nearly constant for both experiments. This figure plots $(e t-e b) / e b$ for each experiment, in which $e t$ and $e b$ are the estimates from the top and bottom, respectively. The error bars show 95\% confidence intervals about the means. As the figure shows, the additional overestimation as a result of viewing from the top rather than the bottom is substantial (roughly $30 \%$ ), but not substantially different across experiments. This is consistent with the idea that the amount of overestimation, in general, varies with size (i.e., falling cost) of the vertical interval estimated, but the degree of overestimation due to relative viewing position is roughly constant.

\section{The Vertical-Horizontal Illusion}

Ultimately, ENT suggests that the descent illusion or other response to falling costs could occur outside of situations with falling costs - as long as doing so imposed fewer costs than failure to overestimate when in the presence of ecologically valid falling costs. This illustrates how ENT may explain the vertical-horizontal illusion (VHI). The classical VHI (see Figure 8) is the tendency to overestimate vertical lines in comparison with equal horizontal lines. The VHI is interesting because we still do not understand why it occurs, even though it is one of the

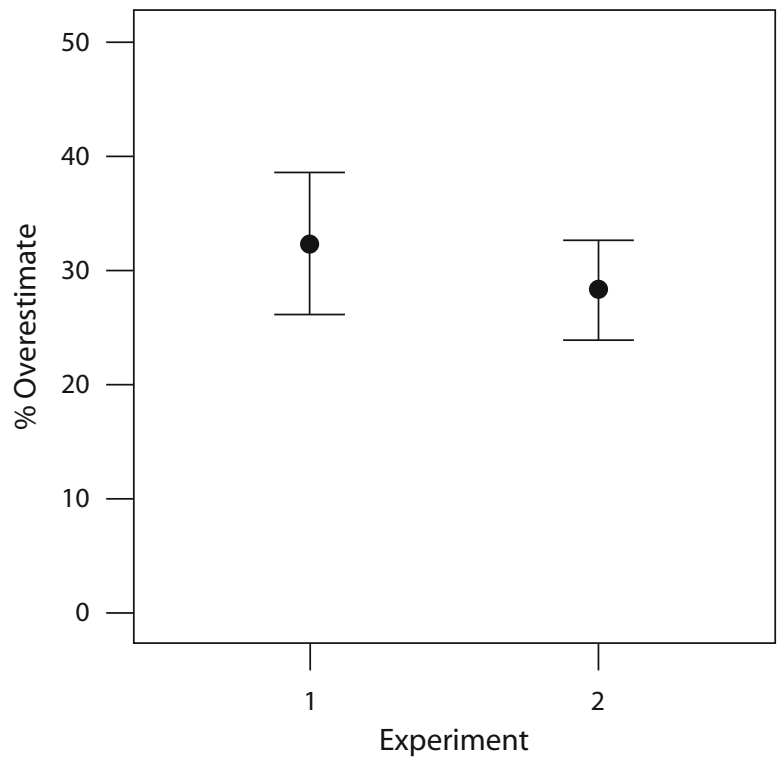

Figure 7. Percent overestimation from the top, in relation to the estimate from the bottom, for each experiment. Plots (et $e b) / e b$, in which $e t$ and $e b$ are the estimates from the top and bottom, respectively. Bars represent $95 \%$ confidence intervals about the means.

oldest psychological phenomena to have been investigated by modern science (Fick, 1851, cited in Finger \& Spelt, 1947; Oppel, 1854, cited in Hicks \& Rivers, 1906), having been researched by the founder of psychological science (Wundt, cited in Winslow, 1933), among others.

ENT suggests that the classical VHI may be a byproduct of mechanisms that evolved to weight navigational choices by the falling costs of vertical navigation (Jackson, 2005). All humans descended from species that suffered falling costs, such as ancestors in common with monkeys. Consistent with ENT, new- and old-world monkeys exhibit the VHI (Dominguez, 1954). ENT also suggests cross-cultural VHI prevalence, because vertical surfaces pose falling costs to all humans. Consistent with ENT, all human populations yet studied display the VHI; this includes populations in environments with minimal receding horizontals, such as the Todas of southern India and the Papuans of New Guinea at the turn of the 20th century (Rivers, 1905), as well as jungle-dwelling Peruvians (Bolton, Michelson, Wilde, \& Bolton, 1975). ENT predicts VHI existence across humans as a species, but ENT also predicts VHI differences within and between

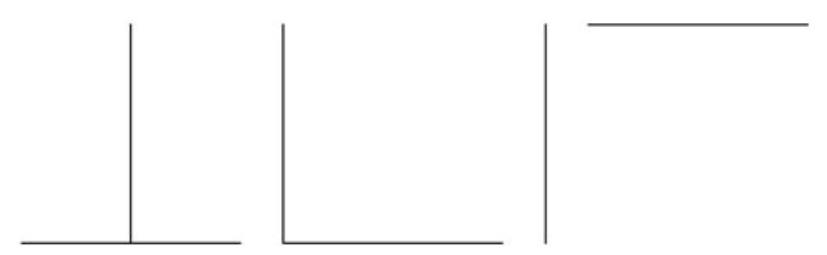

Figure 8. Three common VHI stimuli: upside-down T, L, and separated lines. 
human populations, resulting from variable falling experience and exposure to fall-inducing environments.

Further, ENT suggests that the VHI may increase as viewing conditions become less impoverished and more similar to the conditions in which these adaptations evolved. As mentioned above, Yang et al. (1999) found that participants overestimated a vertical line in natural scenes to a greater percentage when viewing the real stimuli outdoors ( $12 \%$ overestimation) than when viewing photographs ( $2 \%$ overestimation) or drawings $(3 \%$ overestimation) of the same objects. Both Chapanis and Mankin (1967) and the above authors showed that the percentage of magnitude of VHI increased with the perceived object size. Further, Dixon and Proffitt (2002) demonstrated greater VHI when participants viewed an image in a virtual 3-D environment and large, flat virtual movie screen than when they viewed the same image on a virtual computer screen - even though the stimuli subtended the same visual angle across conditions.

Of course, the relation between the classical 2-D VHI and adaptations for 3-D route navigation are thus far speculative. We are currently investigating this potential link with predictions derived from ENT (Jackson \& Cormack, 2006b).

\section{Descent Illusion Mechanism}

These experiments identified a novel perceptual phenomenon, which we term the descent illusion, by using a causal theory without detailing the underlying mechanism. Obviously, some cue or combination of cues produces the descent illusion via some perceptual mechanism, but we are unaware of any obvious cue that could have produced such a large discrepancy between estimates from the top and bottom positions. We are thus far agnostic as to what specific mechanism produces the descent illusion. ${ }^{1}$ The contributions of causal theories such as ENT primarily lie in uniting isolated findings and generating novel, testable hypotheses. These data demonstrate the utility of a causal approach, because researchers now know to look for a mechanism where they previously did not know one existed.

We are currently attempting to determine the cue or combination of cues necessary to produce the descent illusion across both real-world and virtual reality settings (Jackson \& Cormack, 2006a). Given the availability of multiple cues about one's position in space, we find it likely that selection operated on several cues simultaneously. That said, obvious candidates we are exploring include total visual extent of environment, vestibular cues to head orientation, and high-level situational awareness.

\section{Conclusions}

People overestimate the height of a vertical surface, and we have shown that they do so to a dramatically greater extent while positioned at its top. This descent illusion increased as the actual height, and thus the likely falling cost, increased across experiments. Nevertheless, overestimation from the top approximated a relatively constant percentage of the height estimated from the bottom. The height perceived from the top averaged $32 \%$ greater than the height perceived from the bottom, and was as much as $84 \%$ greater than the true height. We predicted the descent illusion by applying a broad, causal theory-ENT - to height perception. ENT focuses on adaptations that arose in response to navigation costs over evolutionary time. ENT applied to height perception directed our investigations to the navigation cost asymmetry of falling, which is much greater for descent than for ascent. This investigation speaks against a view of unitary distance perception and suggests some specialization for both height perception and within different height-perception scenarios.

\section{AUTHOR NOTE}

We thank Emily Bromberg, Joanna Casas, Ashley DePierri, David Dubin, Nancy Egbert, and Ryan Monteiro for collecting data, and Clarke Burnham and two anonymous reviewers for comments. Correspondence concerning this article should be addressed to R. E. Jackson, Department of Psychology, California State University, 333 S. Twin Oaks Valley Rd., San Marcos, CA 92096-0001 (e-mail: russelljackson@mail .utexas.edu).

\section{REFERENCES}

AverY, G. C., \& DAY, R. H. (1969). Basis of the horizontal-vertical illusion. Journal of Experimental Psychology, 81, 376-380.

Avraamides, M. N., Loomis, J. M., Klatzky, R. L., \& Golledge, R. G. (2004). Functional equivalence of spatial representations derived from vision and language: Evidence from allocentric judgments. Journal of Experimental Psychology: Learning, Memory, \& Cognition, 30, 801-814.

Bassett, D. R., Vachon, J. A., Kirkland, A. O., Howley, E. T., DunCAN, G. E., \& Johnson, K. R. (1997). Energy cost of stair climbing and descending on the college alumnus questionnaire. Medicine \& Science in Sports \& Exercise, 29, 1250-1254.

Bolton, R., Michelson, C., Wilde, J., \& Bolton, C. (1975). The heights of illusion: On the relationship between altitude and perception. Ethos, 3, 403-424.

Chapanis, A., \& Mankin, D. A. (1967). The vertical-horizontal illusion in a visually-rich environment. Perception \& Psychophysics, 2, 249-255.

CoHen, H. H., \& LiN, L. (1991). A scenario analysis of ladder fall accidents. Journal of Safety Research, 22, 31-39.

Conroy, G. C. (1997). Reconstructing human origins. New York: Norton.

Da Silva, J. A., \& Dos Santos, R. A. (1984). The effects of instructions on scales for perceived egocentric distance in a large open field. Bulletin of the Psychonomic Society, 22, 189-192.

Dixon, M. W., \& Proffitt, D. R. (2002). Overestimation of heights in virtual reality is influenced more by perceived distal size than by the $2-\mathrm{D}$ versus $3-\mathrm{D}$ dimensionality of the display. Perception, 31, 103-112.

Dominguez, K. E. (1954). A study of visual illusions in the monkey. Journal of Genetic Psychology, 85, 105-127.

Finger, F. W., \& SPELT, D. K. (1947). The illustration of the horizontalvertical illusion. Journal of Experimental Psychology, 37, 243-250.

Foley, J. M., Ribeiro-Filho, N. P., \& Da Silva, J. A. (2004). Visual perception of extent and the geometry of visual space. Vision Research, 44, 147-156.

GiBson, J. J. (1979). The ecological approach to visual perception. Boston: Houghton Mifflin.

Haslam, R. A., \& Bentley, T. A. (1999). Follow-up investigations of slip, trip and fall accidents among postal delivery workers. Safety Science, 32, 33-47.

HICKS, G. D., \& RIVERs, W. H. R. (1906). The illusion of compared horizontal and vertical lines. British Journal of Psychology, 2, 243-260.

Higashiyama, A. (1996). Horizontal and vertical distance perception: The discorded-orientation theory. Perception \& Psychophysics, 58, 259-270. 
Higashiyama, A., \& Ueyama, E. (1988). The perception of vertical and horizontal distances in outdoor settings. Perception \& Psychophysics, 44, 151-156.

Howard, I. P., \& Templeton, W. B. (1966). Human spatial orientation. New York: Wiley.

JACKSON, R. E. (2004). Evolutionary psychology. In W. E. Craighead \& C. B. Nemeroff (Eds.), The concise Corsini encyclopedia of psychology and behavioral science (3rd ed., pp. 341-343). New York: Wiley.

JACKSON, R. E. (2005). Falling towards a theory of the vertical-horizontal illusion. In H. Heft \& K. L. Marsh (Eds.), Studies in perception and action VIII (pp. 241-244). Mahwah, NJ: Erlbaum.

JACKSON, R. E., \& CoRmaCK, L. K. (2006a). Descent illusion mechanism. Manuscript in preparation.

JACKSON, R. E., \& CORMACK, L. K. (2006b). Evolved navigation theory and acrophobia. Manuscript in preparation.

JACKSON, R. E., \& CORMACK, L. K. (2006c). Evolved navigation theory and the environmental vertical illusion. Manuscript submitted for publication.

JACKSON, R. E., \& CORMACK, L. K. (2006d). Evolved navigation theory and the origin of the vertical-horizontal illusion. Manuscript in preparation.

KeEley, L. H. (1996). War before civilization. New York: Oxford University Press.

Mark, L. S., Jiang, Y., Steinbach, K., \& PaAsche, J. (1999). The impact of visual exploration on judgments of whether a gap is crossable. Journal of Experimental Psychology: Human Perception \& Performance, 25, 287-295.

Minetti, A. E., Moia, C., Roi, G. S., Susta, D., \& Ferretti, G. (2002). Energy cost of walking and running at extreme uphill and downhill slopes. Journal of Applied Physiology, 93, 1039-1046.

Rivers, W. H. R. (1905). Observations on the senses of the Todas. British Journal of Psychology, 1, 321-396.
Segall, M. H., Campbell, D. T., \& Herskovits, M. J. (1966). The influence of culture on visual perception. Indianapolis: Bobbs-Merrill.

Somervill, J. W., \& Somervill, C. Z. (1977). Descent behavior of rats and chicks in a cliff and non-cliff situation. Perceptual \& Motor Skills, 45, 391-397.

Svanstrom, L. (1974). Falls on stairs: An epidemiological accident study. Scandinavian Journal of Social Medicine, 2, 113-120.

TEH, K. C., \& AzIz, A. R. (2002). Heart rate, oxygen uptake, and energy cost of ascending and descending the stairs. Medicine \& Science in Sports \& Exercise, 34, 695-699.

Tinetti, M. E., Speechley, M., \& Ginter, S. F. (1988). Risk factors for falls among elderly persons living in the community. New England Journal of Medicine, 319, 1701-1707.

Winslow, C. N. (1933). Visual illusions in the chick. Archives of Psychology, 153, 83 .

Wu, B., OoI, T. L., \& HE, Z. J. (2004). Perceiving distance accurately by a directional process of integrating ground information. Nature, 428, 73-77.

Yang, T. L., Dixon, M. W., \& Proffitt, D. R. (1999). Seeing big things: Overestimation of heights is greater for real objects than for objects in pictures. Perception, 28, 445-467.

\section{NOTE}

1. Our approach, wherein the perception of a surface is perforce influenced by the cost of navigating that surface, might bring to mind Gibson's (1979) notion of affordance. We do not use the term because it carries theoretical implications that we do not necessarily want to convey.

(Manuscript received September 8, 2005; revision accepted for publication March 15, 2006. ) 